Ann. Génét. Sél. anim., I978, 10 (2), 259-270.

\title{
Production de soie et caractéristiques des glandes séricigènes de 13 races de ver à soie (Bombyx mori)
}

\author{
J. M. FAYARD \\ Laboratoire de biométrie, \\ Département de biologie générale et appliquée \\ Université Claude Bernard, Lyon $I$, \\ 69621 Villeurbanne (France).
}

\begin{abstract}
Résumé
Les différences entre races sont étudiées, au niveau cellulaire, sur les glandes séricigènes de ver à soie. Les variations de la production de soie entre treize races de Bombyx mori, sont étudiées en corrélation avec le nombre de cellules, le poids et la teneur en acides nucléiques des glandes séricigènes. Chacune de ces caractéristiques cellulaires, prise séparément, est variable mais ne permet de rendre compte que d'une partie seulement des variations de la synthèse de soie. Les races de vers à soie pourraient se répartir en groupes au sein desquels la sélection serait intervenue préférentiellement sur 1'un de ces caractères.
\end{abstract}

\section{Introduction}

Depuis des milliers de générations, le ver à soie est l'objet d'une sélection intense. $\mathrm{Si}$ on peut admettre que la sélection agit en favorisant 1'association de certains gènes ou de certains groupes de gènes (MAYR, I974), il est cependant difficile d'expliquer comment la production de soie a pu être augmentée de façon aussi considérable.

La sécrétion de la soie résulte du fonctionnement de l'appareil séricigène. Chez Bombyx mori il est constitué par une paire de glandes qui se réunissent en un conduit impair, le tube collecteur, qui débouche sur la presse et la filière. Chaque glande comprend trois parties : un tube sécréteur, un réservoir et un tube conducteur. La soie est constituée de deux protéines : la fibroïne et la séricine. C'est la fibroïne qui est utilisée industriellement. Elle est sécrétée par la partie postérieure de la glande (sécréteur). La séricine est sécrétée par la partie moyenne (réservoir).

Le développement des glandes séricigènes pendant la vie larvaire s'accom- 
pagne d'une évolution particulière des cellules. Leur nombre est fixé à un stade très précoce du développement embryonnaire : elles s'accroissent en taille, 1'ADN est dupliqué de nombreuses fois, mais les cellules ne se divisent pas. Ainsi, elles deviennent géantes avec un taux de ploïdie élevé.

De telles caractéristiques sont certainement, en partie, une conséquence de la sélection. Le ver à soie constitue donc un modèle bien adapté pour juger au niveau cellulaire des effets de la sélection. Des différences quantitatives observées au niveau cellulaire devraient accompagner des différences quantitatives de la production de soie. C'est ce que nous avons cherché à établir par une étude biométrique.

D'une manière générale, trois caractères suffisent pour rendre compte de l'activité sécrétoire d'une glande : le nombre, la taille et 1'activité des cellules. Chez le ver à soie, le nombre des cellules séricigènes est faible; nous avons principalement étudié ce caractère ainsi que sa variabilité (FAYARD, en préparation). Nous nous sommes également intéressé à la taille et à l'activité des cellules. Dans le présent travail, nous rapportons les principaux résultats concernant la corrélation entre l'intensité de la production de soie et les caractéristiques des glandes séricigènes.

\title{
Matériel et méthodes
}

\author{
Ver à soie
}

L'étude a porté sur treize races de vers à soie : trois races à trois mues (75, 74 et 3 mues blanc) et dix races à quatre mues $(200,300,200 \times 300, I 63, I 4 I$, 98, 9 Rose, Ascoli, Chinois blanc et 1'hybride industriel japonais $S_{I 2} 4 \times C_{\text {I24). }}$. Dans le tableau I sont décrites quelques caractéristiques concernant les œufs, les larves et les cocons de chacune des races. Les élevages ont été réalisés à 1'automne. Après une incubation des œufs à $22^{\circ} \mathrm{C}$, les larves sont élevées à $22^{\circ} \mathrm{C}$ et nourries de feuilles fraîches de mûrier Kokuso.

\section{Mesures effectuées}

\section{I. - Dénombrements cellulaires}

En début de troisième âge larvaire, dix mâles et dix femelles sont prélevés au hasard dans les lots expérimentaux. Sur cet échantillon, le nombre des cellules du sécréteur et du réservoir des glandes gauche et droite est estimé.

\section{2. - Poids des glandes}

Comme il n'y a pas multiplication cellulaire au cours de la vie larvaire et que le nombre de cellules est relativement stable, le poids des glandes a été pris comme une estimation de la taille des cellules. Il a été mesuré en début de cinquième âge, avant l'ingestion du premier repas. Le sécréteur et le réservoir de la glande droite de dix individus prélevés au hasard ont été pesés. 
TABLEAU I

Quelques caractéristiques morphologiques des races étudiées

Some morphological characteristics of studied breeds

\begin{tabular}{|c|c|c|c|c|c|}
\hline Races & coque & $\begin{array}{l}\text { OEuf } \\
\text { séreuse }\end{array}$ & $\begin{array}{l}\text { thé- } \\
\text { nce }\end{array}$ & Larve & Cocon \\
\hline $\begin{array}{l}75 \\
\stackrel{0}{\Xi} \\
m \\
m \\
74 \\
3 \text { mues blanc }\end{array}$ & $\begin{array}{l}\text { blanche } \\
\text { blanche } \\
\text { blanche }\end{array}$ & $\begin{array}{l}\text { gris lilas } \\
\text { gris lilas } \\
\text { gris lilas }\end{array}$ & $\begin{array}{l}+ \\
+ \\
+\end{array}$ & $\begin{array}{l}\text { unie, un peu translucent, } \\
\text { sang jaune } \\
\text { unie blanc, sang jaune } \\
\text { unie blanc, sang blanc }\end{array}$ & $\begin{array}{l}\text { rose, allongé en fuseau } \\
\text { chair, allongé en fuseau } \\
\text { blanc, un peu arrondi }\end{array}$ \\
\hline 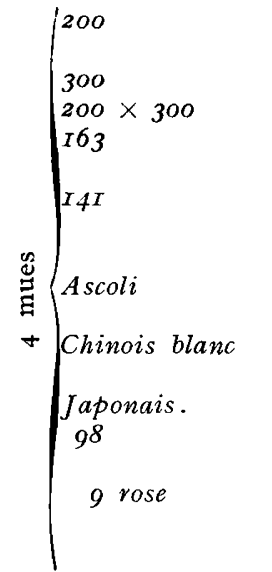 & $\begin{array}{l}\text { blanche } \\
\text { blanche } \\
\text { blanche } \\
\text { blanche } \\
\text { blanche } \\
\\
\text { jaune } \\
\text { gris } \\
\text { jaune } \\
\text { blanche } \\
\text { blanc } \\
\text { jaunâtre } \\
\text { blanc } \\
\text { et jaune }\end{array}$ & $\begin{array}{l}\text { gris lilas } \\
\text { gris lilas } \\
\text { gris lilas } \\
\text { gris lilas } \\
\text { gris lilas } \\
\text { gris lilas } \\
\text { gris lilas } \\
\text { gris lilas } \\
\text { gris } \\
\text { verdâtre } \\
\text { hétéro- } \\
\text { gène }\end{array}$ & $\begin{array}{l}+ \\
+ \\
+ \\
+ \\
-\end{array}$ & $\begin{array}{l}\text { unie, non pigmentée, } \\
\text { non masquée, sang jaune } \\
\text { masquée, sang jaune } \\
\text { sang jaune } \\
\text { masquée, lunules, sang } \\
\text { blanc } \\
\text { moricaud, masque rose, } \\
\text { stars pratiquement ab- } \\
\text { sentes, sang blanc } \\
\text { masquée, z paires de } \\
\text { lunules, sang jaune } \\
\text { blanc unie, sang blanc } \\
\text { masquée, sang blanc } \\
\text { zébrée, polylunulaire, } \\
\text { sang jaune } \\
\text { pigmentation japonaise, } \\
\text { stars à peine marquées, } \\
\text { sang jaune }\end{array}$ & $\begin{array}{l}\text { chair, forme normale } \\
\text { chair, forme normale } \\
\text { chair, forme normale } \\
\text { blanc } \\
\text { blanc } \\
\text { jaune } \\
\text { blanc } \\
\text { blanc } \\
\text { chair } \\
\text { rose }\end{array}$ \\
\hline
\end{tabular}

\section{3. - Dosage des acides nucléiques du sécréteur}

Après dissection, les glandes ont été pesées et conservées à $4^{\circ} \mathrm{C}$, dans une solution d'acide perchlorique à Io p. Ioo. I,es dosages ont été réalisés ultérieurement.

Après broyage dans un potter verre-verre, l'ADN a été extrait par l'acide perchlorique et dosé par la méthode de BuRTon (I956). Une correction a été effectuée pour tenir compte de l'absorption des ARN présents dans la solution. L'ARN a été dosé selon la méthode de MejBAum (I939). Une partie des acides nucléiques est hydrolysée au cours de la conservation de la glande, aussi les dosages ont été réalisés sur les fractions surnageant et culot.

\section{4. - Estimation de la production de soie}

Le filage a été réalisé dans des casiers disposés au-dessus des boîtes d'élevage. Vers le septième jour après la fin du filage, les cocons sont repris et débarrassés 
de la blaze. L'ensemble du cocon, puis la coque seule sont pesés. La richesse soyeuse est calculée suivant la formule :

$$
\text { R.S. }=\frac{\text { Poids de la coque }}{\text { Poids du cocon }} \times \text { roo }
$$

Chaque lot expérimental comprend dix mâles et dix femelles.

Dans certains cas on a également déterminé le taux de fibroïne, après dissolution de la séricine dans une solution de $\mathrm{Na}_{2} \mathrm{CO}_{3} 0,5$ p. Ioo chauffée à $90{ }^{\circ} \mathrm{C}$.

\section{Résultats}

L'ensemble des mesures effectuées sur chaque race a été regroupé dans le tableau 2. I,es corrélations ont été calculées sur des moyennes par race et sexe.

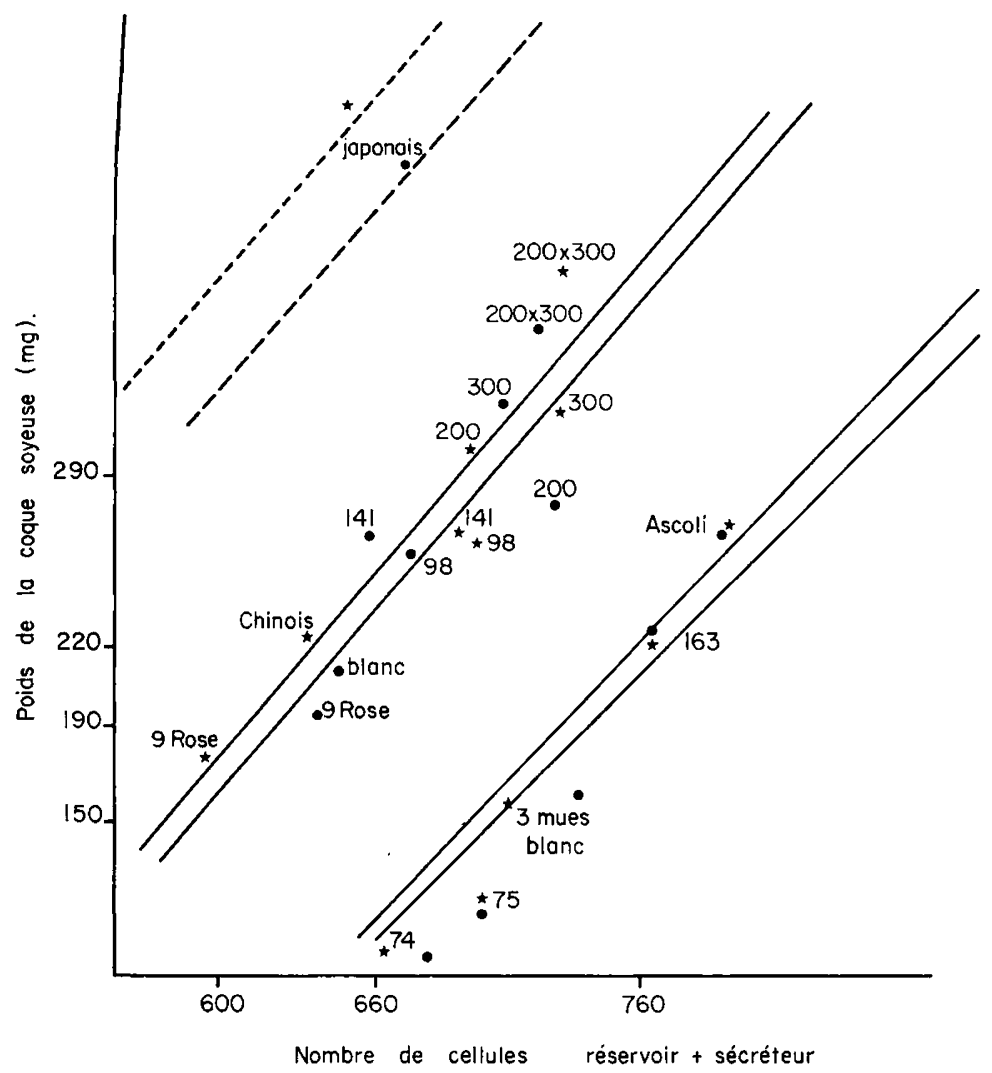

FIG. I. - Variations du poids de la coque en fonction du nombre des cellules de la glande séricigène.

Variation of shell weight function of the number of cells of silk gland.

males (males)

$\star$ femelles (females) 


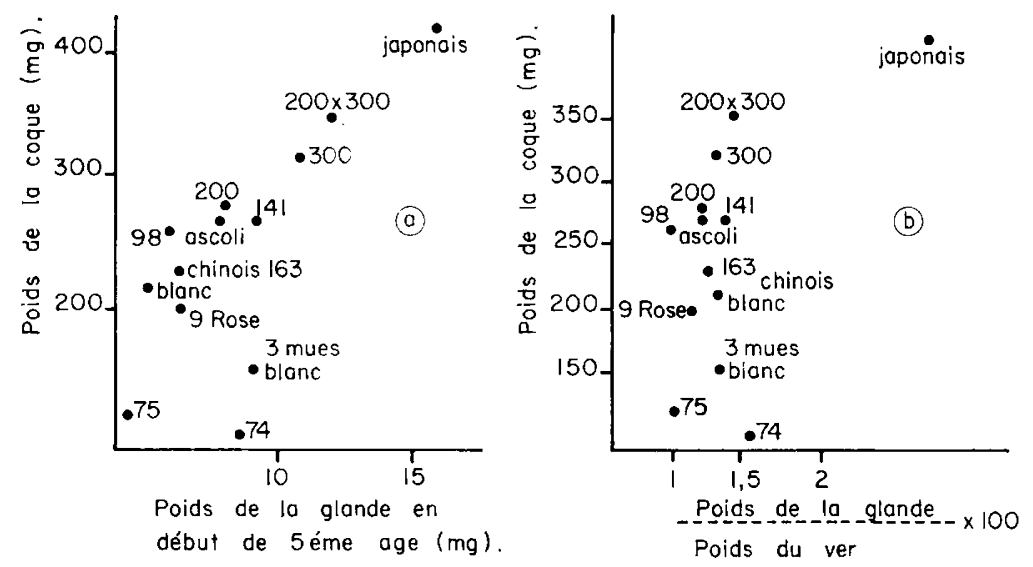

FIG. 2. - Variations du poids de la coque, chez les mâles, en fonction:

a) du poids de la glande en début de $5^{\mathrm{e}}$ âge,

b) du poids relatif de la glande en début de $5^{\mathrm{e}}$ âge.

Des graphes semblables sont obtenus pour les femelles.

Variations of shell weight function:

a) of the weight of the gland at the beginning of age 5

b) of the relative weight of the gland at the beginning of age 5 .

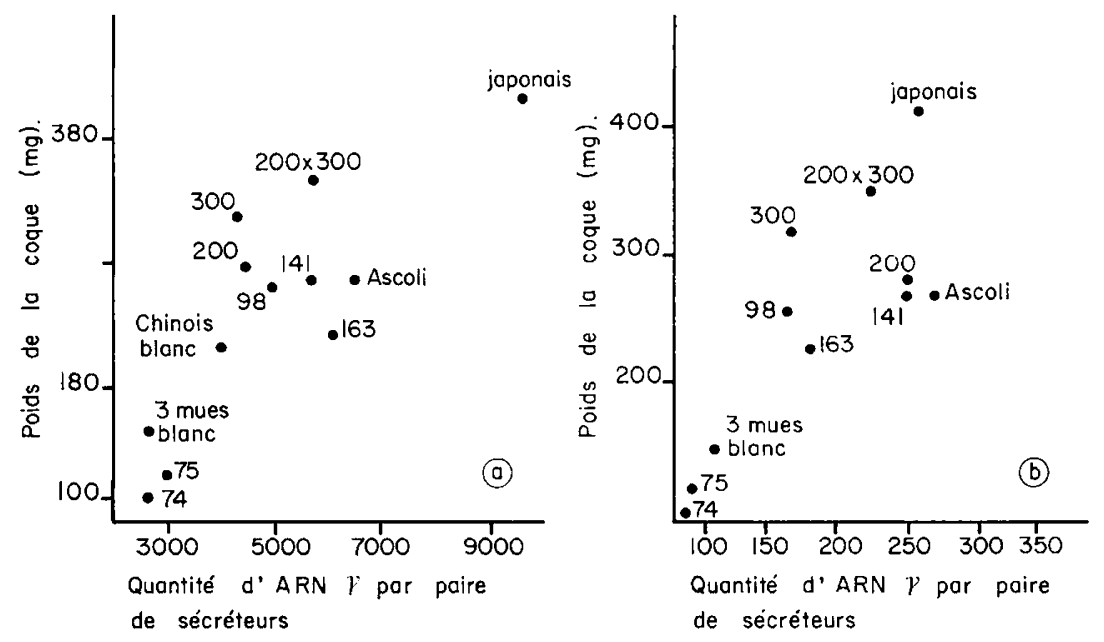

Frg. 3. - Variations du poids de la coque soyeuse chez les mâles, en fonction:

a) de la quantité d'ARN du tube sécréteur,

b) de la quantité d'ADN du tube sécréteur.

Variations of weight of silk shells in the male function:

a) of the amount of RNA in the secretory tube,

b) of the amount of DNA in the secretory tube. 
TABLE

Récapitulatif des résultats obtenus. Les moyennes sont accompagnées Summary of resu

\begin{tabular}{|c|c|c|c|c|c|c|c|c|c|}
\hline \multirow{3}{*}{\multicolumn{2}{|c|}{ Races }} & \multicolumn{2}{|c|}{$\begin{array}{l}\text { Nombre } \\
\text { cellules }\end{array}$} & \multicolumn{4}{|c|}{$\begin{array}{l}\text { Poids de la glande } \\
\text { en début de } 5^{\text {e âge }}\end{array}$} & \multicolumn{2}{|c|}{$\begin{array}{l}\text { Acides nucléiques } \\
\text { du sécréteur } 5^{\mathrm{e}} \text { â }\end{array}$} \\
\hline & & \multirow{2}{*}{$\begin{array}{l}\text { Sécré- } \\
\text { teur }\end{array}$} & \multirow{2}{*}{$\begin{array}{l}\text { Réser- } \\
\text { voir }\end{array}$} & \multicolumn{2}{|c|}{ Sécréteur } & \multicolumn{2}{|c|}{ Réservoir } & $\mathrm{ADN}$ & ARN \\
\hline & & & & $\mathrm{mg}$ & $\%$ & $\mathrm{mg}$ & $\%$ & $\gamma /$ paire & écréteu \\
\hline & 5 & 477 & 249 & 2,33 & 0,28 & 3,57 & 0,43 & 223 & 5709 \\
\hline 200 & $\sigma$ & 43 & I 8 & 0,15 & 0,03 & 0,30 & 0,05 & 33 & I 519 \\
\hline$x$ & 이 & $4^{8 I}$ & $25^{2}$ & $3, \mathrm{I} 7$ & 0,30 & 4,29 & $0,4 \mathrm{I}$ & 207 & 7218 \\
\hline \multirow[t]{2}{*}{$3^{\circ 0}$} & $\sigma$ & 46 & $2 \mathrm{I}$ & $\mathrm{I}, 03$ & 0,07 & $\mathrm{I}, 5 \mathrm{I}$ & 0,12 & 28 & I 157 \\
\hline & t) & 476 & $24^{8}$ & I,97 & 0,23 & 3,43 & $0,4 \mathrm{I}$ & 167 & 4363 \\
\hline \multirow[t]{4}{*}{$3^{\circ 0}$} & $\sigma$ & 36 & I9 & 0,79 & 0,07 & 1,39 & 0,12 & I 4 & 814 \\
\hline & 아 & 477 & 253 & 2,31 & 0,24 & 3,65 & $0,3^{8}$ & $\mathrm{I} 8 \mathrm{I}$ & 5617 \\
\hline & $\sigma$ & $3 \mathrm{I}$ & I6 & $0,3^{8}$ & 0,03 & 0,64 & 0,06 & & \\
\hline & $\pi$ & $4^{80}$ & 249 & 1,60 & 0,24 & 2,36 & 0,35 & 252 & 4544 \\
\hline \multirow[t]{4}{*}{200} & $\sigma$ & 34 & I 5 & 0,30 & 0,05 & 0,53 & 0,08 & & \\
\hline & q & 454 & 243 & 1,68 & 0,22 & $2,8 \mathrm{I}$ & 0,37 & 218 & 5326 \\
\hline & $\sigma$ & I 5 & I 8 & 0,22 & 0,04 & 0,30 & 0,03 & & \\
\hline & $\pi$ & 445 & 230 & 1,79 & 0,30 & 2,13 & 0,35 & 162 & 5177 \\
\hline \multirow[t]{4}{*}{98} & $\sigma$ & 27 & 12 & 0,39 & 0,04 & 0,47 & 0,04 & & \\
\hline & $q$ & 459 & 240 & $\mathrm{I}, 54$ & 0,26 & 1,76 & 0,23 & I 87 & 4563 \\
\hline & $\sigma$ & $3 I$ & I I & 0,13 & 0,03 & 0,06 & 0,03 & & \\
\hline & శ. & $5^{\circ 4}$ & 264 & $I, 73$ & 0,33 & $2,3 I$ & 0,45 & I 79 & 6000 \\
\hline \multirow[t]{4}{*}{ I63 } & $\sigma$ & 24 & 14 & 0,49 & 0,06 & $0,4^{8}$ & 0,06 & & \\
\hline & 이 & 509 & 260 & $\mathrm{I}, 78$ & 0,29 & I,95 & $0,3 \mathrm{I}$ & I97 & 6807 \\
\hline & 0 & $3 I$ & I4 & $0, \mathbf{I} 8$ & 0,02 & 0,21 & 0,03 & & \\
\hline & $\sigma^{\pi}$ & 429 & $23^{\circ}$ & $\mathrm{I}, 9 \mathrm{I}$ & 0,29 & 2,62 & $0,3^{8}$ & 249 & 4767 \\
\hline \multirow[t]{4}{*}{ I $4 \mathrm{I}$} & $\sigma$ & 33 & 14 & I, I 3 & 0,01 & 0,72 & 0,09 & 57 & 564 \\
\hline & of & 450 & $24 \mathrm{I}$ & 1,36 & $0, \mathrm{I} 8$ & $2, \mathrm{OI}$ & 0,27 & & \\
\hline & $\sigma$ & 36 & Io & 0,49 & 0,05 & 0,84 & 0,09 & & \\
\hline & $0^{x}$ & 399 & 239 & $\mathrm{I}, 54$ & 0,27 & $\mathrm{I}, 70$ & 0,30 & & \\
\hline \multirow{4}{*}{ Rose } & $\sigma$ & 28 & 27 & $0,5 \mathrm{I}$ & 0,07 & 0,62 & 0,09 & & \\
\hline & of & 362 & 233 & 1,83 & 0,26 & $\mathrm{I}, 73$ & 0,39 & & \\
\hline & $\sigma$ & 39 & 23 & 0,60 & 0,06 & 0,92 & 0,10 & & \\
\hline & a & 428 & 218 & $\mathrm{I}, 52$ & 0,36 & 1,83 & 0,46 & & \\
\hline \multirow[t]{2}{*}{ Chinois } & $\sigma$ & 37 & I 8 & 0,87 & 0,15 & 0,60 & 0,13 & & \\
\hline & 우 & 422 & 209 & $\mathrm{I}, 46$ & 0,35 & $\mathrm{I}, 49$ & 0,36 & & \\
\hline \multirow[t]{2}{*}{ Blanc } & $\sigma$ & 37 & 14 & 0,46 & $0, \mathbf{I}$ I & 0,20 & 0,04 & & \\
\hline & $\sigma^{x}$ & 445 & 228 & $4, \mathrm{I} 4$ & 0,63 & & & $26 I$ & 9679 \\
\hline Japonais & $\sigma$ & I 8 & I I & $0,5 \mathrm{I}$ & 0,07 & & & $2 \mathrm{I}$ & I 656 \\
\hline & o & 424 & 227 & 4,86 & 0,59 & & & 234 & 9892 \\
\hline & $\sigma$ & 25 & 9 & 0,59 & 0,09 & & & & \\
\hline & $d$ & 524 & 271 & 1,29 & 0,19 & 2,54 & $0,3^{8}$ & 266 & $757 \mathrm{I}$ \\
\hline Ascoli & $\sigma$ & 34 & 19 & 0,37 & 0,04 & 0,49 & 0,04 & 55 & 849 \\
\hline & 이 & 524 & 274 & $\mathrm{I}, 5^{8}$ & 0,22 & 2,27 & $0,3 \mathrm{I}$ & 273 & 5171 \\
\hline & $\sigma$ & 28 & I 6 & $0,4 \mathrm{I}$ & 0,06 & 0,26 & 0,03 & & \\
\hline & $\sigma^{*}$ & 479 & $25^{\circ}$ & 2,27 & 0,35 & 3,39 & 0,49 & I IO & 2723 \\
\hline 3 mues & $\sigma$ & 28 & I I & 0,89 & 0,15 & I, 16 & 0,19 & I6 & 566 \\
\hline Blanc & 이 & $45 I$ & 247 & 3,33 & 0,35 & 4,44 & 0,46 & & \\
\hline & $\sigma$ & 26 & I 3 & 0,83 & 0,09 & 0,95 & 0,09 & & \\
\hline & $0^{x}$ & $4 \mathrm{I} 4$ & 224 & 1,36 & 0,30 & $I, 51$ & 0,34 & 90 & $282 \mathrm{I}$ \\
\hline 75 & $\sigma$ & 36 & Io & 0,66 & 0,09 & 0,54 & 0,09 & 9 & 595 \\
\hline & 우 & 413 & 225 & $\mathrm{I}, 43$ & 0,27 & 1,70 & 0,32 & 109 & 3229 \\
\hline & $\sigma$ & 36 & 12 & 0,67 & 0,09 & 0,77 & 0,09 & & \\
\hline & $\sigma^{a}$ & 452 & 226 & 2,17 & $0,4^{\circ}$ & $2, \mathrm{II}$ & 0,39 & 85 & 2622 \\
\hline 74 & $\sigma$ & $3 I$ & II & 0,28 & 0,05 & $0,3^{\circ}$ & 0,06 & 17 & 718 \\
\hline & o & 433 & 229 & $2,3^{8}$ & 0,37 & $2,3^{8}$ & 0,37 & 145 & $326 I$ \\
\hline & $\sigma$ & 30 & I I & 0,79 & 0,08 & 0,70 & 0,08 & & \\
\hline
\end{tabular}


'́cart-type, lorsqu'elles ont été obtenues à partir de 5 répétitions et plus

\begin{tabular}{|c|c|c|c|c|c|c|c|c|c|}
\hline \multicolumn{3}{|c|}{$\begin{array}{l}\text { Production } \\
\text { de soie }\end{array}$} & \multicolumn{7}{|c|}{$\begin{array}{l}\text { Valeurs des caractères } \\
\text { par cellule de sécréteur }\end{array}$} \\
\hline $\mathrm{g}$ & $\begin{array}{l}\text { Coque } \\
\mathrm{mg}\end{array}$ & $\begin{array}{c}\text { Fibroïne } \\
\text { mg }\end{array}$ & $\begin{array}{l}\text { Fibroïne } \\
\text { mg/cell. }\end{array}$ & $\begin{array}{c}\text { ADN } \\
\gamma / \text { cell. } \\
\text { secr. }\end{array}$ & $\begin{array}{c}\text { ARN } \\
\gamma / \text { cell. } \\
\text { secr. }\end{array}$ & 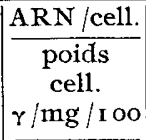 & $\begin{array}{c}\text { Mol. Fib. } \\
\text { Nbre de } \\
\text { gènes } \\
\times \text { 10 } \\
\end{array}$ & $\begin{array}{l}\frac{\text { Prot. }}{\text { ARN }} \\
\times \text { Ioo }\end{array}$ & $\frac{A R N}{A D N}$ \\
\hline $\begin{array}{l}\mathrm{I}, 87 \\
0,16\end{array}$ & $\begin{array}{r}35^{\circ} \\
39\end{array}$ & $\begin{array}{r}232 \\
25\end{array}$ & 0,24 & 0,23 & 6 & 12,3 & 13 & $4, \mathrm{I}$ & 26 \\
\hline $\begin{array}{l}2,46 \\
0,19\end{array}$ & $\begin{array}{r}374 \\
36\end{array}$ & $\begin{array}{r}235 \\
25\end{array}$ & 0,24 & 0,22 & 7,5 & I I , 4 & I 4 & 3,3 & 35 \\
\hline $\begin{array}{l}1,62 \\
0,17\end{array}$ & $\begin{array}{r}3^{\mathrm{I}} 7 \\
3^{8}\end{array}$ & $\begin{array}{r}196 \\
22\end{array}$ & $0,2 \mathrm{I}$ & $\mathrm{o}, \mathrm{I} 8$ & 4,6 & I I, I & I 5 & 4,5 & 26 \\
\hline $\begin{array}{l}2,00 \\
0,14\end{array}$ & $\begin{array}{r}3 \mathrm{I} 6 \\
4^{\circ}\end{array}$ & $\begin{array}{r}203 \\
25\end{array}$ & $0,2 \mathrm{I}$ & 0,19 & 5,9 & $\mathrm{I} 2,2$ & I 4 & 3,6 & $3^{I}$ \\
\hline $\begin{array}{l}1,65 \\
0,19\end{array}$ & $\begin{array}{r}279 \\
7 \mathrm{I}\end{array}$ & $\begin{array}{r}\text { I94 } \\
25\end{array}$ & 0,20 & 0,26 & 4,8 & 14,2 & Io & 4,3 & I 8 \\
\hline $\begin{array}{l}2,00 \\
0,13\end{array}$ & $\begin{array}{r}298 \\
24\end{array}$ & $\begin{array}{r}191 \\
13\end{array}$ & $0,2 \mathrm{I}$ & 0,24 & 5,9 & I 5,9 & I I & 3,6 & 24 \\
\hline $\begin{array}{l}1,62 \\
0,09\end{array}$ & $\begin{array}{r}258 \\
26\end{array}$ & $\begin{array}{r}\text { I } 77 \\
22\end{array}$ & 0,20 & $0, \mathrm{x} 8$ & 5,5 & I 4,5 & I4 & 3,4 & 32 \\
\hline $\begin{array}{l}\mathbf{1}, 99 \\
0,199\end{array}$ & $\begin{array}{r}273 \\
37\end{array}$ & $\begin{array}{r}173 \\
27\end{array}$ & 0,19 & 0,20 & 5,0 & $\mathrm{I}_{4}, 8$ & I 2 & 3,8 & 24 \\
\hline $\begin{array}{l}I, 36 \\
0,11\end{array}$ & $\begin{array}{r}227 \\
40\end{array}$ & $\begin{array}{r}162 \\
32\end{array}$ & 0,16 & $0, \mathrm{I} 8$ & 5,4 & $\mathrm{I} 7,3$ & I I & 2,7 & 34 \\
\hline $\begin{array}{l}\mathrm{I}, 86 \\
0,08\end{array}$ & $\begin{array}{r}225 \\
27\end{array}$ & $\begin{array}{r}\mathrm{I} 6 \mathrm{I} \\
\mathrm{I} 7\end{array}$ & $0, \mathrm{I} 6$ & O, I9 & 5,7 & $19, \mathrm{I}$ & 10 & 2,4 & 35 \\
\hline $\begin{array}{l}\mathrm{r}, 65 \\
0,17\end{array}$ & $\begin{array}{r}267 \\
30\end{array}$ & $\begin{array}{r}189 \\
23\end{array}$ & 0,22 & $0,29]$ & 5,5 & $\mathrm{I} 2,5$ & Io & 3,9 & 19 \\
\hline 2,05 & 270 & I9O & $0,2 \mathrm{I}$ & & & & & & \\
\hline $\begin{array}{l}0,22 \\
\mathrm{I}, \mathrm{I} 8\end{array}$ & $\begin{array}{r}49 \\
196\end{array}$ & $\begin{array}{r}35 \\
\times 34\end{array}$ & $\mathrm{o}, \mathrm{I} 7$ & & & & & & \\
\hline 0,09 & I 7 & $\mathrm{I} 2$ & & & & & & & \\
\hline $\begin{array}{l}I, 37 \\
\text { o,2 I }\end{array}$ & $\begin{array}{r}\text { I } 75 \\
2 \mathrm{I}\end{array}$ & $\begin{array}{r}12 \mathrm{I} \\
24\end{array}$ & 0,17 & & & & & & \\
\hline $\begin{array}{l}\mathrm{I}, 07 \\
0,08\end{array}$ & $\begin{array}{r}210 \\
18\end{array}$ & $\begin{array}{r}147 \\
6\end{array}$ & 0,17 & & & & & & \\
\hline I, 46 & 226 & $\begin{array}{r}10 \\
158\end{array}$ & 0,19 & & & & & & \\
\hline 0,17 & I9 & I6 & & & & & & & \\
\hline $\begin{array}{l}\mathrm{r}, 79 \\
\mathrm{o}, 20\end{array}$ & $\begin{array}{r}412 \\
28\end{array}$ & $\begin{array}{r}289 \\
23\end{array}$ & 0,32 & 0,30 & тo,8 & I I ,7 & $r_{4}$ & 3,0 & 37 \\
\hline $\begin{array}{l}2,24 \\
0,24\end{array}$ & $\begin{array}{r}437 \\
\mathrm{I} 7\end{array}$ & $\begin{array}{r}292 \\
18\end{array}$ & 0,34 & 0,28 & I I, 7 & 10,2 & I 6 & 3,0 & 42 \\
\hline I,68 & 267 & I69 & 0,16 & 0,25 & 7,3 & 29,3 & 8 & 2,2 & 29 \\
\hline $\begin{array}{l}0,03 \\
\mathrm{I}, 95\end{array}$ & $\begin{array}{r}25 \\
268\end{array}$ & $\begin{array}{r}22 \\
162\end{array}$ & 055 & $0>06$ & & 164 & & & \\
\hline o,I 7 & 29 & $x 7$ & 0,15 & 0,26 & 4,9 & & 7 & $3, \mathrm{I}$ & 19 \\
\hline $\begin{array}{l}0,92 \\
0,09\end{array}$ & $\begin{array}{r}\text { I } 5 \text { I } \\
\text { I }\end{array}$ & $\begin{array}{r}106 \\
22\end{array}$ & $0, \mathrm{II}$ & $0, \mathrm{I} \mathrm{I}$ & 8,8 & 6,0 & 12 & 3,9 & 25 \\
\hline r, 10 & $\mathrm{I}_{4} \mathrm{I}$ & 100 & 0, I I & & & & & & \\
\hline 0,15 & 26 & r 7 & & & & & & & \\
\hline $0,9^{\circ}$ & I 16 & 77 & 0,09 & O,I I & 3,4 & 10,4 & I I & 2,7 & $3 I$ \\
\hline $\begin{array}{l}\mathrm{O}, \mathrm{I} 2 \\
\mathrm{I}, \mathrm{IO}\end{array}$ & $\begin{array}{r}\mathrm{I} 7 \\
\mathrm{I} \mathbf{I}\end{array}$ & $\begin{array}{l}\text { I4 } \\
7 \mathrm{I}\end{array}$ & 0,09 & 0,13 & 3,9 & II, 3 & 8 & 2,2 & 30 \\
\hline 0,16 & 29 & $2 \mathrm{I}$ & & & & & & & \\
\hline 0,70 & 98 & 65 & 0,07 & 0,09 & 2,9 & 6,0 & Io & 2,5 & $3 \mathrm{I}$ \\
\hline $\begin{array}{l}0,08 \\
0,87\end{array}$ & $\begin{array}{l}\text { I3 } \\
99\end{array}$ & $\begin{array}{r}3 \\
69\end{array}$ & 0,08 & $0, \mathrm{I} 7$ & 3,8 & 6,9 & 6 & $2, \mathrm{I}$ & 23 \\
\hline 0,05 & 9 & 7 & & & & & & & \\
\hline
\end{tabular}




\section{I. - Relation entre production de soie et nombre de cellules}

La figure i montre la corrélation entre le nombre total des cellules du sécréteur et du réservoir et le poids de la coque. Les coefficients de corrélation calculés pour l'ensemble des valeurs, chez les mâles et chez les femelles, ne sont pas significativement différents de zéro. Les races étudiées peuvent se répartir en trois groupes : le polyhybride japonais; les races à trois mues, r63 et Ascoli; les autres races constituant le troisième groupe.

Dans les deuxième et troisième groupes, la corrélation entre quantité de soie produite par la glande et le nombre de cellules peut être estimée par le coefficient de corrélation de rang de Spearman (SIEGEL, I956) :

$$
\begin{array}{r}
\text { mâles : } r_{\mathrm{s}}=0,93 \quad(p<0,0 \mathrm{I}) \\
\text { femelles }: r_{\mathrm{s}}=0,90 \quad(p=0,05)
\end{array}
$$

Pour ces deux groupes, nous avons calculé le coefficient de corrélation linéaire et l'équation de la droite de régression : ( $\mathrm{Y}=$ quantité de soie en $\mathrm{mg}, \mathrm{X}=$ Nombre de cellules).

$$
\begin{aligned}
& r \quad \text { équation de la droite de régression } \\
& \begin{array}{lll}
\text { Groupe } 2 & \text { के } & 0,88 \\
& \text { o } & 0,93
\end{array} \\
& \text { Groupe } 3 \text { o } 0,93 \\
& \text { 우 } 0,98 \\
& \begin{array}{l}
\mathrm{Y}=\mathrm{I}, 20 \mathrm{X}-55^{2} \\
\mathrm{Y}=\mathrm{I}, \mathrm{I} 7 \mathrm{X}-5^{23} \\
\mathrm{Y}=\mathrm{I}, 05 \mathrm{X}-585 \\
\mathrm{Y}=\mathrm{I}, 03 \mathrm{X}-566
\end{array}
\end{aligned}
$$

Malgré le faible nombre de répétitions, les coefficients sont différents de zéro. La corrélation semble plus élevée chez les femelles. Les coefficients de régression ne diffèrent pas entre les groupes ni entre les sexes. Une étude de la corrélation entre le nombre de cellules du sécréteur et le poids de fibroïne ou entre le nombre de cellules du réservoir et le poids de séricine ne permet pas d'améliorer la précision de ces résultats.

\section{2. - Corrélation entre production de soie et poids des glandes (fig. 2)}

Pour les races à quatre mues, nous avons calculé les coefficients de corrélation ainsi que l'équation des droites de régression pour chaque sexe et pour la production globale de soie et la production de fibroïne.

$$
\begin{aligned}
& r \quad \text { Equation de la droite de régression } \\
& \text { o } 0,94 \quad \mathrm{Y}=0,53 \mathrm{X}+36,44 \quad \mathrm{Y}=\text { poids de la coque (mg); } \\
& \text { 우 } 0,72 \quad \mathrm{Y}=0,29 \mathrm{X}+\mathrm{I} 39 \quad \mathrm{X}=\text { poids de la glande (mg) } \\
& \text { ô } 0,78 \quad \mathrm{Y}=0,75 \mathrm{X}+47 \quad \mathrm{Y}=\text { poids de fibroïne }(\mathrm{mg}) \\
& \text { 아 } 0,63 \quad \mathrm{Y}=0,36 \mathrm{X}+\text { IIo } \mathrm{X}=\text { poids du sécréteur }(\mathrm{mg})
\end{aligned}
$$

Les coefficients de corrélation et de régression sont plus élevés chez les mâles. Ils varient aussi suivant le caractère. 


\section{3. - Corrélation entre production de soie et acides nucléiques du sécréteur (fig. 3)}

Les quantités d'ARN sont très variables. Pour l'ensemble des races nous avons calculé le coefficient de corrélation et l'équation de la droite de régression chez les mâles et les femelles.

\begin{tabular}{cccc} 
& $r$ & \multicolumn{1}{c}{ Droite de régression } & \\
0 & 0,80 & $\mathrm{Y}=0,036 \mathrm{X}+65,27$ & $\mathrm{Y}=$ poids de la coque (mg) \\
o & 0,87 & $\mathrm{Y}=0,046 \mathrm{X}+6,226$ & $\mathrm{X}=\underset{\text { quantité d'ARN }(\gamma / \text { paire }}{\text { de sécréteurs })}$
\end{tabular}

De même pour l'ADN :

\begin{tabular}{|c|c|}
\hline$r$ & Droite de régression \\
\hline o 0,82 & $\mathrm{Y}=\mathrm{I}, \mathrm{I} 4 \mathrm{X}+37, \mathrm{I} 6$ \\
\hline 우 0,70 & $Y=1,61 X-46,67$ \\
\hline
\end{tabular}

\section{Discussion}

L'ensemble de nos résultats montre que la liaison entre la production de soie et les mesures réalisées sur les glandes séricigènes n'est pas simple. Le poids des glandes et les teneurs en acides nucléiques semblent assez bien corrélés avec la production de soie, mais il convient d'être prudent pour l'interprétation de telles corrélations. La comparaison de races très différentes peut permettre de révéler l'existence d'une corrélation masquée, au niveau individuel, par l'effet d'autres facteurs. Une telle corrélation peut n'être en quelque sorte qu'un artefact à l'évolution parallèle de deux caractères indépendants.

D'autre part, les résultats obtenus sur toutes les races montrent que la production de soie et le nombre de cellules sont peu corrélés. Cependant, sur la figure $I$, on peut reconnaitre trois groupes de races pour lesquels il semble exister une relation linéaire entre la production de soie et le nombre de cellules séricigènes. Ainsi, nous sommes conduit à penser que les races se répartissent en groupes au sein desquels c'est le nombre de cellules de la glande séricigène qui détermine la variabilité de la production de soie. En tête de chaque groupe considéré, se trouvent des races qui ont été très améliorées, ce qui ne saurait être une coïncidence. Ise nombre de cellules pourrait ainsi, dans certaines conditions, être le caractère modifié par la sélection.

De grandes différences physiologiques et génétiques font que, pour 1'ensemble des caractères étudiés, les races à troies mues se séparent toujours des autres races. Ceci est vrai en particulier pour le poids des glandes. La corrélation entre ce caractère et la production de soie est plus élevée lorsqu'on ne considère que les races à quatre mues (fig. 2a). En début de $5^{\mathrm{e}}$ âge, la quantité de soie présente dans la lumière de la glande est négligeable. Le poids constitue donc bien une estimation de la taille des cellules et la production de soie pourrait dépendre de ce caractère. Elle n'est cependant pas corrélée avec la valeur relative du poids des plandes. Ainsi, pour la production totale de soie et poids relatif de la glande (secréteur et réservoir), $r=-0, \mathrm{I} 4$ pour les mâles et $r=0,054$ pour les femelles. Tout se passe comme si l'importance de la sécrétion était déterminée plus par des carac- 
téristiques propres de la glande que par ses relations avec le reste de l'organisme.

L'examen des figures $3 a$ et $3 b$ montre que la teneur de la glande en acides nucléiques et la production de soie varient dans le même sens.

Les teneurs en $A D N$ varient de 85 à I27 $\gamma$ par paire de sécréteurs. Les seules variations du nombre de cellules ne permettent pas de rendre compte de telles variations. De même, il n'est pas possible de répartir les races étudiées en deux groupes différant par le nombre de cycles de duplication de 1'ADN, même si la teneur en $\mathrm{ADN}$ chez les races à troies mues semble correspondre à un cycle de division de moins que pour les races à quatre mues. Pour rendre compte des valeurs intermédiaires (races 98, I6 3 par exemple), 1'hypothèse la plus simple consiste à admettre que le nombre de cycles n'est pas le même pour toutes les cellules. Par dosage des acides nucléiques, GAGE (I974) et par autoradiographie, Gri,ot et a1. (I 968) ont estimé que chaque noyau du sécréteur subit en moyenne I 7 à 18 endoduplications. Les cycles ne se produisent pas simultanément dans toutes les cellules, mais une synchronisation se réalise à l'occasion de chaque mue. Ainsi, on peut supposer qu'au cours du $5^{\mathrm{e}}$ âge, toutes les cellules du sécréteur seraient le siège d'au moins deux cycles de divisions. Pour une certaine proportion de cellules, il y aurait un cycle supplémentaire. La valeur de cette proportion pouvant dépendre de facteurs génétiques (différences entre races) ou même environnementaux.

Pour une même quantité d'ADN, nous constatons que la production de soie varie (races 98 et 300, Ascoli et Japonais, I4I et $200 \times 300$ par exemple). L'activité des glandes ne peut donc pas être simplement reliée à la quantité d'ADN. Le graphe $3^{b}$ montre que la corrélation avec 1'ARN est meilleure. Une étude quantitative a déjà été abordée par KuRATA et al. (en préparation) qui ont mis en évidence sur des races également très différentes, une relation linéaire entre la quantité de soie filée et la teneur en ARN présente dans la glande le $6^{\mathrm{e}}$ jour du $5^{\mathrm{e}}$ âge larvaire. PRUDhomme (I976) a également montré que l'évolution de la synthèse

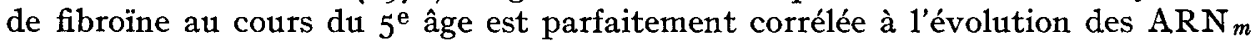
au cours de la même période.

La considération de l'ensemble des mesures réalisées sur les glandes séricigènes montre qu'on ne peut pas rendre compte des variations de la production de soie par les variations d'un seul caractère. La sécrétion de soie résulte de phénomènes complexes qui mettent en jeu de nombreux caractères. Ainsi, on peut concevoir qu'au niveau cellulaire, les mécanismes d'action de la sélection varient selon les races. Le graphe I illustre bien cette hypothèse selon laquelle il y a plusieurs réponses possibles à une pression de sélection. L'amélioration d'un caractère ne se produit d'ailleurs pas de manière continue au cours des générations : elle passe le plus souvent par des paliers successifs. Ceux-ci pourraient correspondre à des " cibles " différentes qui seraient atteintes successivement au cours d'une sélection. Ainsi, sur le graphe I, pour chaque groupe de races considéré, c'est l'augmentation du nombre de cellules qui aurait permis l'augmentation de la production de soie. Le passage d'un groupe à un autre résultant des variations d'un autre caractère.

On peut considérer que la quantité totale de soie produite par les glandes séricigènes est fonction du nombre de cellules et de la production de chaque cellule, elle-même dépendant de caractères que nous avons cherché à préciser. Dans le tableau 2 sont indiquées, pour le sécréteur, les valeurs des différents caractères, rapportées à chaque cellule.

Pour II races, c'est-à-dire pour les races à trois mues et les races à quatre mues, nous avons pu faire une régression multiple entre la production de soie 
et les variables explicatives : quantité d'ARN et d'ADN en fin de $5^{\text {e âge. La }}$ variable la mieux corrélée avec la production de soie est la quantité d'ADN. Ce caractère détermine en quelque sorte la puissance de synthèse de la cellule. Puisque, pour une même quantité d'ADN, la production de soie par cellule peut être très différente, c'est que l'efficacité de la synthèse est très différente selon les races. GAGE (I974) et RASCH (I974) ont estimé à IO $^{-6} \mathrm{~g}$ la taille du génome diploïde chez Bombyx mori. Sachant qu'il n'y a ni amplification génique, ni duplication préférentielle du gène Fibroïne (GAGE, I976), nous pouvons déduire de la quantité d'ADN par cellule, le nombre moyen de gènes Fibroïne par cellule (tabl. 2). De même, le poids moléculaire de la Fibroïne étant estimé à 400 ooo daltons (PRUDhomme, I976), on peut calculer le nombre moyen de molécules de Fibroïne synthétisées par cellule du sécréteur. Ces deux valeurs nous permettent d'estimer un rendement global pour la synthèse de fibrö̈ne:

$$
\mathrm{R}=\frac{\text { Nombre de molécules de fibroïne }}{\text { Nombre de gènes fibroïne }}
$$

Ce rendement comprend au moins deux composantes relatives à la transcription et à la traduction et quie nous avons estimées respectivement par les rapports $\frac{\mathrm{ARN}}{\mathrm{ADN}}$ et $\frac{\text { poids de fibroïne }}{\mathrm{ARN}}$ (tab1. 2). Les variations de $\mathrm{R}$ montrent des différences importantes dans le fonctionnement de la proteosynthèse. L'examen du tableau I montre que ces variations peuvent provenir soit de la transcription, soit de la traduction.

Les différences observées au niveau de la transcription peuvent provenir de diverses causes. La polyplö̈die engendre une forme particulière d'amplification parmi tous les gènes présents dans la cellule, combien sont actifs simultanément? La vitesse de transcription peut également varier avec le nombre de chaînes d'ADN initialisées en même temps ou même, avec la vitesse de lecture proprement dite. Enfin, on peut envisager des variations de la durée de vie des ARN.

De la même manière, le rendement de la traduction peut dépendre des caractéristiques du système moléculaire : vitesse de lecture des $A R N_{m}$, nombre de chaînes protéiques synthétisées simultanément sur un même $\mathrm{ARN}_{m}$. Remarquons que la synthèse de la fibroïne s'effectue au niveau de l'ergastoplasme et que le transport de la molécule vers la lumière de la glande fait intervenir le Golgi. Ainsi, le rendement de la synthèse peut dépendre de la place disponible dans la cellule, et on peut envisager de simples contraintes physiques. La taille de la cellule jouant alors un rôle de régulation sur la synthèse protéique.

Ainsi, notre travail nous a conduit à supposer que la sélection n'est pas intervenue de la même manière pour toutes les races. Au niveau cellulaire, les trois caractères, nombre, taille et teneur en acides nucléiques des cellules sont susceptibles de varier et de rendre compte d'une partie des variations de la production de soie. Il conviendrait donc de choisir les critères de sélection en fonction des caractéristiques des glandes séricigènes et compte tenu de 1'histoire probable de la sélection. On peut par exemple envisager qu'une sélection sur le nombre de cellules pourrait être efficace chez les japonais alors qu'il conviendrait mieux de sélectionner la race Ascoli pour la taille des cellules. 


\section{Summary \\ Silk production and characteristics of silkglands of 13 breeds of silkworm (Bombyx mori)}

Differences between races are analysed at cellular level on the silkglands of Bombyx mori. The fluctuations of silk production between thirteen races of silkworm are studied in correlation with silkgland cell number, weight and nucleic acids content. Each of the these cellular characteristics, taken separately, is variable, but it only reflects a part of the variations of silk synthesis. It is suggested that the different races can be divided into groups in which the selection had occured preferably on one of the these characteristics.

\section{Références bibliographiques}

BURTON K., I956. A study of the conditions and mechanism of the diphenyl amine reaction for the colorimetric estimation of deoxyribonucleic acid. Biochem., 5, 315-323.

GAGE L.: P., r974. The Bombyx mori genome: analysis by DNA reassociation kinetics. Chromosoma, 45, $27-42$.

GaGe L. P., MANning B. F., I976. Determination of the multiplicity of the silk fibroin gene and detection of fibroin gene-related DNA in the genome of Bombyx mori. J. Mol. Biol., 101, $327-348$.

Grilor S., DAILliE J., I968. Rapport entre la mue et la synthèse d'ADN dans la glande séricigène dù ver à soie. C. R. Acad. Sci., 266, 2295.

Mayr E., I974. In Populations, especes et évolution. Hermann, Paris.

Mejbaum W., I939. Estimation of small amounts of pentose especially in derivatives of adenylic acid. Hoppe-Seyler's $Z$. Physiol. Chem., 258, iा7-I20.

Pruphomme J. C., I976. Contribution à l'étude de la biosynthèse de la fibroïne dans la glande séricigène de Bombyx mori. Thèse doct. sci. nat., Lyon.

RASch F. M., I974. The DNA content of sperm and hemocyte nuclei of the silkworm Bombyx mori. Chromosoma, 45, I.

SIEGEL S., I956. Non parametric statistics for the behavioral sciences. Mc Graw Hill Book Company, Inv. New-York, Toronto, London. 